\title{
Perspectives of adolescents, parents, and teachers on barriers and facilitators of physical activity among school-age adolescents: a qualitative analysis
}

El-Ammari Abdelghaffar ${ }^{1 *}$ D, El Kazdouh Hicham, Bouftini Siham', El Fakir Samira ${ }^{1}$ and El Achhab Youness ${ }^{1,2}$

\begin{abstract}
Background: Physical activity levels are low among adolescents in Morocco; however, the influences on physical activity behavior of adolescents have not yet been explored in a qualitative study. Here, we explored potential social-ecological barriers and facilitators of physical activity in Moroccan adolescents with the goal of developing a successful intervention program aimed at improving their physical activity level.

Methods: For this study, we conducted 17 focus group discussions (100 participants, composed of 56 adolescents, 26 parents, and 18 teachers from two middle schools in Taza city, Morocco). Discussions during focus groups were facilitated by a semi-structured interview guide. Guide questions were underpinned by the social-ecological model as a theoretical framework. Data analysis was carried out by two coders using thematic analysis.

Results: We found that barriers and facilitators of physical activity in adolescents are organized into six themes that belong to different levels of the social-ecological model. Three themes belonged to the intrapersonal level (perceived motivating and limiting factors, physical activity awareness, and time constraints), two themes were classified into the interpersonal/cultural level (social support and gender and cultural norms), and one theme belonged to the environmental level (access to opportunities). Most of the themes were at the individual level, with each theme including both barriers and facilitators.

Conclusions: Adolescent participation in physical activity can be facilitated or hampered by many factors. Results from the focus group discussions showed that these factors belonged to different levels of the social-ecological model, but most were at the individual level. Our findings have several implications. First, they may offer suggestions for a tailored intervention program aimed at improving adolescent physical activity. Second, they can improve quantitative research by enriching the battery of questions of physical activity instruments (e.g., a question related to physical disability). Third, the proposed thematic map can contribute to understanding interactions and causal pathways in the social-ecological model.
\end{abstract}

Keywords: Perspectives, Barriers, Facilitators, Adolescents, Physical activity, Morocco, Qualitative study

\footnotetext{
* Correspondence: abdelghaffar.elammari@usmba.ac.ma

${ }^{1}$ Laboratory of Epidemiology, Clinical Research and Community Health,

Faculty of Medicine and Pharmacy, University Sidi Mohamed Ben Abdellah,

Fez, Morocco

Full list of author information is available at the end of the article
}

(c) The Author(s). 2019 Open Access This article is distributed under the terms of the Creative Commons Attribution 4.0 International License (http://creativecommons.org/licenses/by/4.0/), which permits unrestricted use, distribution, and reproduction in any medium, provided you give appropriate credit to the original author(s) and the source, provide a link to the Creative Commons license, and indicate if changes were made. The Creative Commons Public Domain Dedication waiver (http://creativecommons.org/publicdomain/zero/1.0/) applies to the data made available in this article, unless otherwise stated. 


\section{Background}

Adolescence is a period of biological and social transition, in which future patterns of adult health are established [1, 2]. Although most adolescents are perceived as healthy, important behavioral risk factors emerge during adolescence, and these behaviors can lead to disorders in adulthood [2, 3]. A decline in physical activity (PA) marks this period of life [4]. Worldwide, physical inactivity has become the fourth leading cause of global mortality. In 2008, more than 5.3 million of the 57 million deaths that occurred worldwide were attributable to physical inactivity [5]. Lack of PA is related to several noncommunicable diseases (including heart diseases, diabetes, and cancer), as well as other health problems [5, 6]. Maintaining adequate levels of PA among adolescents could substantially improve physical and mental health (as measured by, for example, cardiorespiratory and muscular fitness, bone health, cardiovascular metabolic health biomarkers, body composition, and psychosocial outcomes) [7-11]. Although burdens associated with physical inactivity are widely recognized, more than $80 \%$ of adolescents worldwide are physically inactive based on the World Health Organization-defined threshold [12]. According to their definition, to achieve health benefits, adolescents should accumulate at least $60 \mathrm{~min}$ of moderate to vigorous intensity PA every day [6]. Therefore, interventions to improve adolescent PA are needed, especially because PA habits adopted during adolescence can track to adulthood [13].

To develop an effective intervention, what is first needed is a better understanding of why adolescents (do not) engage in PA [14]. To reach this goal, ecological frameworks are particularly suitable because they consider the plurality and the complex interplay between the potential influences on PA [15]. Ecological models are a comprehensive approach that span factors affecting PA at multiple levels: the intrapersonal level (biological, psychological); the interpersonal/cultural, organizational, and physical environment level (built, natural); and the policy level (laws, rules, regulations, codes) [15].

Evidence from quantitative designs has shown that engagement of adolescents in PA is influenced by factors belonging to different levels of the ecological model, with determinants of adolescent PA mainly shown to be within the intrapersonal level. These determinants include age, sex, socioeconomic status, and psychological and biological factors [16-18]. In addition, several consistent influencing factors have been shown to be within the social and physical environments (including social support from family and friends and access to opportunities for PA) [16, 1821]. PA research has been often dominated by quantitative approaches; however, qualitative studies have recently been rising. There are some complementary components between quantitative and qualitative approaches [22, 23]. But, qualitative research can offer additional understanding on key factors that facilitate "facilitators" and hamper "barriers" to adolescent participation in PA, as well as result in suggestions from adolescent and other participants on how to implement a successful intervention to improve PA levels in adolescents [24]. Findings from qualitative studies have shown that the key factors that influence adolescent PA are within the individual level (including enjoyment, perceived benefits, and motivation) [25-29] or within the interpersonal and cultural levels (e.g., social support) [25, 27-31] or even within the environmental level (e.g., accessibility and availability of PA opportunities) [25-29, 31].

Like many countries [12, 32], physical inactivity rates among Moroccan adolescents is alarming. In 2016, the Global School-based Student Health Survey reported that only $11.0 \%$ of Moroccan school-age adolescents are active [33]. Many factors are responsible for this situation; some of them are common with other counties, but others are specific to our Arab-Muslim culture society which seem particularly discouraging of PA [34]. The practice of PA is not a valued habit in Morocco for a variety of physical and cultural reasons [35]. There is a lack of parental support to PA, partly because parents favor educational success over exercise for their children [34, 35]. The practice of PA is known to manage weight, but in almost all countries in the Arab world, people tend to tolerate fatness, and there were no social sanctions against adiposity [36]. On the contrary, female plumpness is culturally preferred in some Moroccan ethnic group and even represents a symbol of beauty and prosperity [37]. Men are more active than women because of conservative social norms and cultural restrictions on outdoor activities and exercise for women [34, 35]. Although Morocco is undergoing an epidemiological transition [38], data on factors influencing PA among adolescents are lacking. Influencing factors of PA have never been explored among Moroccan adolescents using a qualitative design. Here, we conducted a qualitative study to identify potential social-ecological barriers and facilitators of adolescent PA.

\section{Methods \\ Study design}

To investigate social-ecological factors influencing adolescent PA, we collected qualitative data from participants in Taza city, a medium-sized city (207,984 inhabitants) in north-central Morocco [39], from February to July 2016. Specifically, semi-structured focus groups (FG) were asked about their perspectives and experiences related to factors that influence adolescent PA. Because triangulation of data sources guarantees the credibility of results from qualitative studies [24, 40], we included three types of participants: adolescents, parents, and teachers. The study was approved by the Faculty of Medicine and Pharmacy of Casablanca Research Ethics Committee and by the National Control Commission for the Protection of Personal 
Data (A-RS-193-2015) and authorized by The Provincial Directorate of Education Ministry in Taza. Informed consent was obtained from all parents and teachers. Adolescent consent forms were signed by their parents, but an additional verbal consent was also given by adolescent participants. Participant privacy was protected through anonymous and voluntary participation.

This qualitative study is part of a larger underway research project [41], which has a major goal of developing and implementing an intervention that supports a healthy lifestyle among adolescents. To reach this goal, potential factors influencing risk behaviors (e.g., PA and nutrition) were first identified through a mixed methods approach involving a quantitative study [42].

\section{Participants}

Participants were recruited from two middle schools (disadvantaged and advantaged per socioeconomic level) that participated in the previous quantitative study [42]. From each school, 14- to 16-year-old students were invited to participate in FG discussions. This age group of students was selected because their curriculum includes topics related to health risk behaviors. Teachers were of the three school subjects that are concerned with health risk behaviors: "Physical Education," "Life and Earth Sciences," and "Islamic Education."

Participant recruitment was significantly facilitated by directors and the general surveyor of schools. Adolescent, parent, and teacher participants first received an information letter specific for their group type. The letter provided a comprehensive explanation of study objectives and processes and contained an informed consent to sign. Second, after the participant informed consent was received, researchers, participants, and school officials selected an appropriate date and setting to conduct FGs. Unlike quantitative studies, the number of participants was not previously calculated, and sampling continued until data saturation was reached.

\section{Data collection}

We chose the FG method because it encourages interactive discussions between participants [43]. Discussions were conducted by a moderator and an observer who are already trained to conduct FG discussions. The moderator managed the discussions with participants while the observer noted nonverbal cues (e.g., mimics, gestures, silence) and group interactions that seemed useful to understanding nonverbal participation. All FG discussions were conducted in the Arabic language, the mother tongue of the participants and interviewers.

The FG discussions followed a semi-structured interview that began with an introduction, followed by an opening question, and then open-ended questions that served to map further discussions to identify barriers and facilitators of adolescent PA (Table 1). Our FG guide, which was developed using the social-ecological framework as a theoretical framework [15], was pilot tested with a convenient sample of each type of participant and refined before definitive use. The total number of FGs was determined by the saturation principle.

FGs were homogeneous regarding sex to overcome possible bias and to provide an opportunity for possible gender-specific factors to emerge [29]. In both schools, FG discussions were conducted in a suitable room where participants felt most comfortable and which allows a good quality audio recording. Participants also gave permission to record and use their anonymous quotes in research publications.

\section{Data analysis}

Audio tapes from the discussion were fully transcribed and organized into separate datasets for adolescents, parents, and teachers. Relevant field notes such as nonverbal cues were also included in the transcribing process. All datasets and notes were analyzed by the coders using thematic analysis method of Braun and Clarke, which is a method for identifying, analyzing, and

Table 1 Focus group discussion steps

\begin{tabular}{|c|c|c|c|}
\hline & Adolescents & Parents & Teachers \\
\hline Step 1: Introduction & \multicolumn{3}{|c|}{$\begin{array}{l}\text { After the moderator and observer introduced themselves, the moderator stated the study objective and clarified anonymity, and } \\
\text { some consigns to conduct discussion. Permission to record the interview was also obtained. Finally, PA was defined in detail. }\end{array}$} \\
\hline $\begin{array}{l}\text { Step 2: Opening } \\
\text { question }\end{array}$ & $\begin{array}{l}\text { AQ1: Each of you describe your PA and } \\
\text { then tell us how much time you should } \\
\text { devote to PA each day to stay healthy? }\end{array}$ & $\begin{array}{l}\text { PQ1: Please, can you tell us how much } \\
\text { time your children can devote to PA } \\
\text { each day to stay healthy? }\end{array}$ & $\begin{array}{l}\text { TQ1: Please, what can you tell us } \\
\text { about the PA level of adolescents } \\
\text { today? }\end{array}$ \\
\hline $\begin{array}{l}\text { Step 3: Open-ended } \\
\text { questions to identify } \\
\text { barriers and facilitators } \\
\text { of adolescent PA }\end{array}$ & $\begin{array}{l}\text { AQ2: Please tell us the factors that } \\
\text { help you to do PA? Give any factors, } \\
\text { regardless of their nature. } \\
\text { AQ3: Please tell us the factors that } \\
\text { impede you to do PA? Give any } \\
\text { factors that exist, regardless of } \\
\text { their nature. }\end{array}$ & $\begin{array}{l}\text { PQ2: Please tell us the factors that help } \\
\text { your children engage in PA? Give any } \\
\text { factors that seem relevant. } \\
\text { PQ3: Please tell us the factors that prevent } \\
\text { your children from engaging in PA? Here } \\
\text { too, please tell us any factors, regardless } \\
\text { of their nature. }\end{array}$ & $\begin{array}{l}\text { TQ2: Please, what are the barriers to } \\
\text { PA among adolescents? Give any } \\
\text { factors that seem relevant. } \\
\text { TQ3: How do you evaluate the role } \\
\text { that the school plays in adolescent PA? }\end{array}$ \\
\hline
\end{tabular}


reporting themes within data [44]. Themes are patterns which capture something interesting about the data in relation to the research question [44]. Coders followed one-by-one the different steps proposed by Braun and Clarke: become familiar with the data, generate initial codes, search for themes, review themes, define themes, and write-up [44]. Coders started reading and re-reading the interview transcripts to become familiar with the data, took notes, and jot down early impressions. Then, open coding was used; that means we did not have preset codes but developed and modified the codes as we worked through the coding process. In addition, the coding process included consideration of our research question. So, this was an inductive-deductive thematic analysis. The next step was to search for themes; once the initial codes were generated, we started to categorize and combine them to form overarching themes. Themes were predominately at the semantic level, i.e., they are identified within the explicit or surface meanings of the data. After developing the initial themes, they were continually reviewed, created, and discarded using an iterative process. Thus, we constantly moved back and forth between the selected extracts from the data and the entire dataset to check if the themes make sense and account for all the coded extracts and the entire data set to assess the applicability of themes. Finally, each theme was defined, labeled, and analyzed. A thematic map was also established; it shows the organization of themes according to different levels of the social-ecological model and possible interactions between themes.

To ensure transparency and reliability, all transcripts were coded thoroughly by two researchers (the first and the second author of this paper) independently. Regular meetings with the research team were conducted to discuss and agree on any outstanding questions. The analysis team discussed their coding and interpretation of the transcripts in detail, and any coding differences were discussed and resolved to refine codes and identify key themes from the data. We translated into English the quotes used to illustrate themes and subthemes. No software was used in the data analysis.

\section{Results}

A total of 100 people participated in 17 FGs. The estimated point of saturation was observed after 8 FGs of adolescents ( $n=56,50 \%$ boys), 5 FGs of parents $(n=26$, $81 \%$ males), and 4 FGs of teachers $(n=18)$. Each FG discussion lasted between 50 and $80 \mathrm{~min}$. Additional descriptive sample characteristics are presented in Table 2.

After data analysis, six main themes regarding the perceived barriers and facilitators of adolescent PA were identified: (1) perceived motivating and limiting factors, (2) PA awareness, (3) perceived time constraints, (4) social support, (5) cultural and gender norms, and (6) access to
Table 2 Participant characteristics

\begin{tabular}{ll}
\hline Adolescents ( $n=56)$ & 7 \\
No. of participants per group & 50 \\
Sex (\% boys) & 14 to 16 \\
Age range (years) & 3 rd grade of middle school \\
Grade level & \\
Perceived family income & 5.36 \\
$\quad$ Low (\%) & 94.94 \\
$\quad$ Average (\%) & 0.0 \\
$\quad$ High (\%) & \\
Education level of parents & 4.0 \\
$\quad$ Fathers (\% illiterate) & 36.36 \\
$\quad$ Mothers (\% illiterate) & \\
Parents ( $n=26$ ) & 5 to 6 \\
No. of participants per focus group & 19.23 \\
Sex (\% female) & 30 to 60 \\
Age range (years) & 0 \\
Education level (\% illiterate) & \\
Teachers ( $n=18$ ) & 40 to 50 \\
No. of participants per focus group & \\
Age range (years) &
\end{tabular}

opportunities. Further descriptions of each theme and subtheme, as well as informative quotes in support of our results, are shown in Table 3 . Themes 1, 2, and 3 were intrapersonal-level themes, themes 4 and 5 were interpersonal/cultural-level themes, and theme 6 was the single environmental-level theme. Figure 1 presents a thematic map that shows the organization of themes according to different levels of the social-ecological model and possible interactions between themes.

\section{Theme 1: Perceived motivating and limiting factors}

This theme refers to internal motivations and restrictions toward adolescent PA. It comprises three subthemes: enjoyment and competition, perceived health benefits, and perceived limiting factors.

\section{Subtheme 1: Perceived enjoyment and competition}

Expressions used by both male and female adolescents to support this subtheme included "Sport pleases me a lot," "I'm enjoying by doing sport," and "I exercise to entertain myself." Having fun was a strong facilitator of adolescent PA. Some adolescents expressed a strong desire to exercise, with PA allowing them an outlet from daily routines and the ability to share enjoyment with friends. Parents and teachers also confirmed these statements. Competition was perceived as a facilitator for PA by some participants, which were almost exclusively boys. 


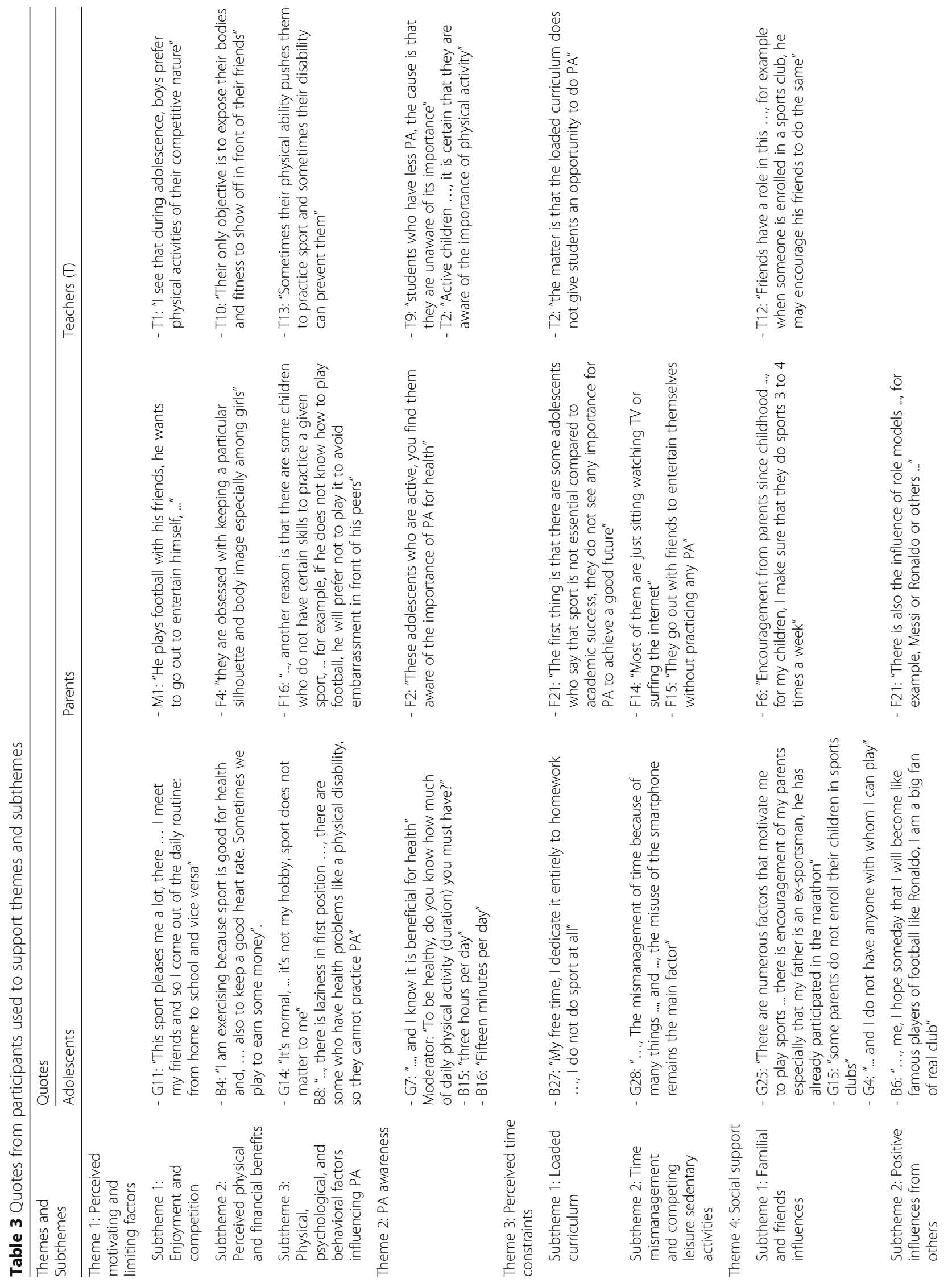




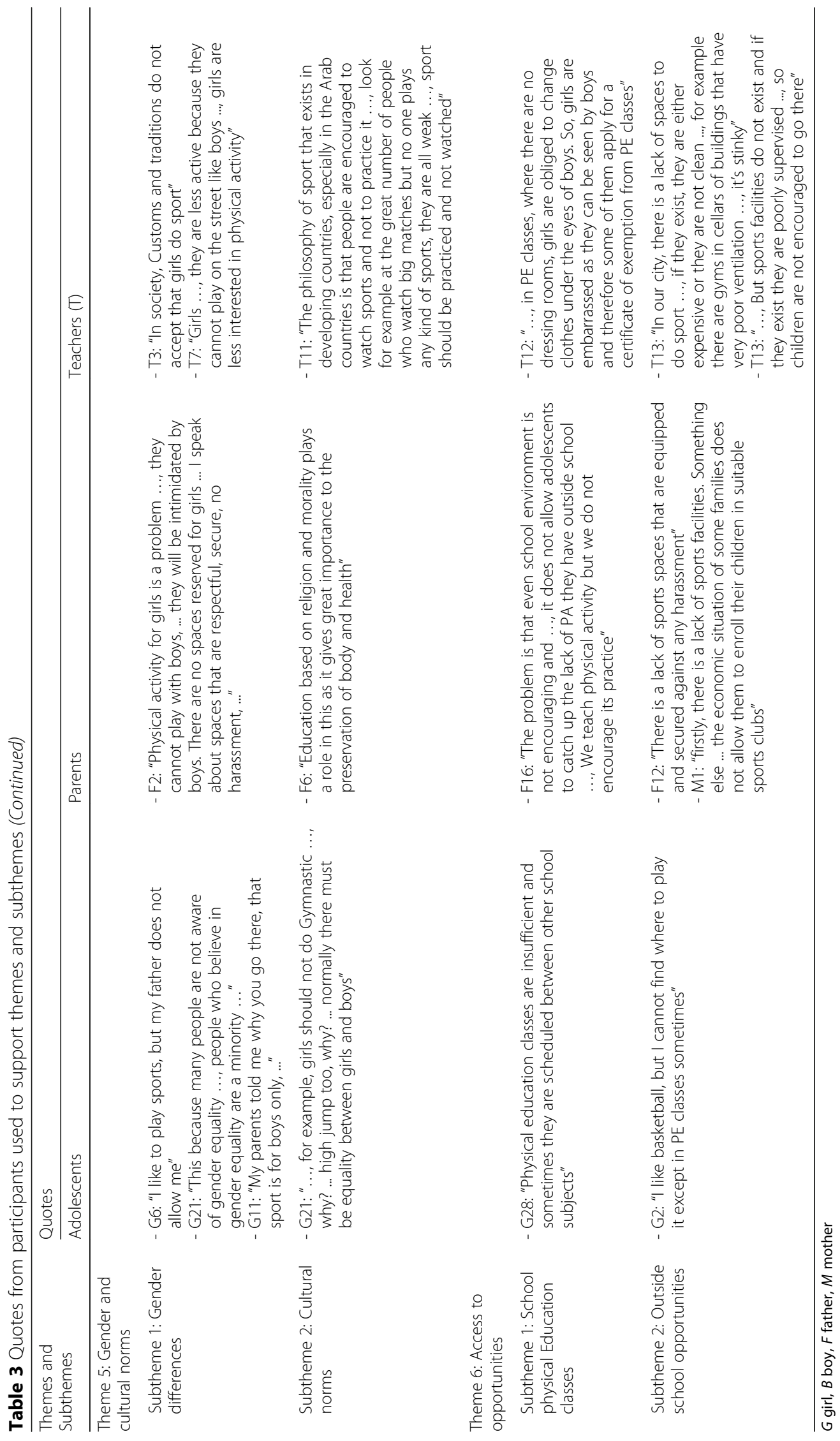




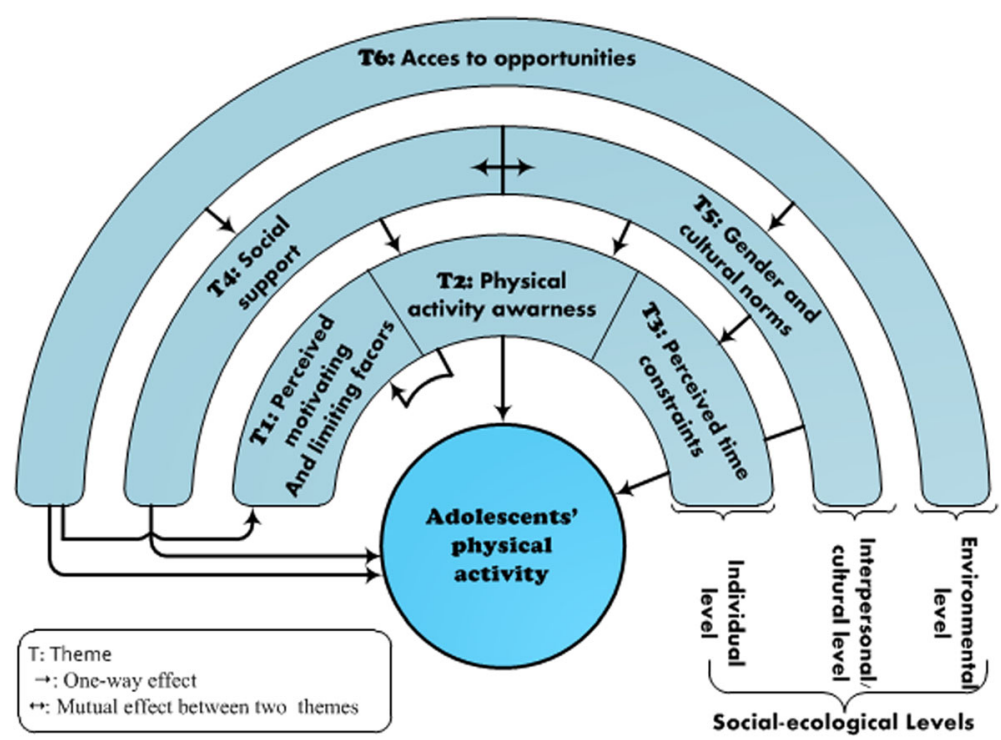

Fig. 1 Thematic map showing identified themes and potential interactions between them

\section{Subtheme 2: Perceived health and financial benefits}

Participants expressed that preserving physical and mental health in adolescence and in adulthood was a reason to engage in PA. Weight control, self-defense, physical appearance, and showing off muscles (among boys) to friends were also reasons for adolescents to engage in PA. Surprisingly, some adolescents practiced football for financial objective, contributing a sum of money that would be ultimately awarded to the winning team.

\section{Subtheme 3: Physical, psychological, and behavioral factors influencing $P A$}

Participants stated that these physical, psychological, and behavioral factors were overwhelmingly barriers to adolescent PA and included the presence of a physical disability, poor fitness, lack of skills in sports, lack of interest toward PA, laziness, timidity, and fear of accidents. Participants also mentioned that adolescents who smoke or use drugs are unmotivated to be active. Some parents and teachers had a different perspective and did not consider this subtheme as barriers to PA. Instead, they expressed that having some physical skills, having willingness, not having a disability, or being away from substance use were facilitators of PA.

\section{Theme 2: Physical activity awareness}

This theme could be both a barrier and a facilitator of PA, depending on the level of adolescent awareness regarding PA. Awareness included having knowledge of adequate PA duration and intensity and the benefits on health (e.g., physical and psychological health, disease prevention). Participants stated that media, family, and school play a role in raising PA awareness, although some participants argued that awareness played only a modest role. Parents and teachers highlighted that young people were often disinterested toward advice from others.

\section{Theme 3: Time constraints}

This theme comprised two subthemes, both being barriers to PA: (1) overloaded curriculum and (2) time mismanagement and competing leisure sedentary activities.

\section{Subtheme 1: Overloaded curriculum}

Many participants mentioned that overloaded curriculum and homework greatly reduce adolescent leisure time, thus reducing the participation of adolescents in PA. In addition, some adolescents favored educational achievements versus participation in PA.

\section{Subtheme 2: Time mismanagement and competing leisure sedentary activities}

Mismanagement of time and not devoting a place to PA in the daily schedule of adolescents represented a barrier to PA. Many participants stated that they waste substantial time in front of a screen (including television, smartphone, tablet, or laptop), performing household tasks (for girls), or spending time with friends.

\section{Theme 4: Social support}

Active adolescents had support from family members, which was also confirmed by parents and teachers. Parental support included encouragement and incentive to exercise, enrolment in sport clubs especially at an early age, and parental modeling. In addition, a family atmosphere without problems or conflicts supported adolescent PA. Support from friends was also stated by participants as a 
facilitator of adolescent PA. In addition, some adolescents practiced PA just to meet and spend time with friends.

Many participants highlighted that lack of support from parents, family, or friends has a negative influence on adolescent participation in PA. Lacking PA awareness in the family, not having friends, having friends who are inactive, or having friends with other interests or negative interests (e.g., substance use) keep adolescents away from practicing PA.

Some participants expressed that role models could also include successful national sports figures who may inspire adolescents to engage in sports.

\section{Theme 5: Gender and cultural norms}

\section{Subtheme 1: Gender differences}

Girls, parents, and teachers explicitly reported that girls are particularly underprivileged in PA engagement compared with boys. Indeed, some girls stated that their parents prevented them from practicing PA. In addition, boys tended to take over neighborhood spaces with sports activities, especially football. Some girls expressed that it was shameful for them to participate in outside PA. Girls expressed spending almost all their leisure time at home, doing homework or helping their mothers in housework chores.

Girls and parents stated that there is a lack of appropriate spaces specific for girls that are secure, safe, and well supervised. There is a fear of being intimidated, harassed, or even assaulted. A final opinion that was emphasized by some teachers is that girls are less interested in PA than boys.

\section{Subtheme 2: Cultural norms}

According to the participants, some sports are perceived to be valid only for boys. They also argued that gender equality was not yet implemented in the society. Some teachers referred to customs and traditions as barriers to PA participation among girls. In addition, some parents and teachers stated that even if religion encourages participation in PA, a culture of PA is not common in the society. FG discussions also showed that young people watch sports incessantly through media outlets without practicing any kind of it.

\section{Theme 6: Access to opportunities}

\section{Subtheme 1: School physical education classes}

Physical education (PE) classes are sometimes the only opportunity to practice PA. According to some participants, PE classes are insufficient ( $2 \mathrm{~h}$ per week), in fact, not meeting the PA threshold recommended for adolescents. In addition, PE classes face several difficulties. Participants state that PE classes are forbidden during bad weather; some adolescents stated that playgrounds were insufficient and unpractical. In addition, participants stated that PE classes are scheduled between other school subjects. PE classes at school also necessitated dressing rooms, which are often unavailable. Participants reported that girls were unwilling to participate in PE classes when there are no dressing rooms. Because girls are embarrassed as they can be seen by boys, they often apply for exemption from PE classes.

\section{Subtheme 2: Outside school opportunities}

Most participants indicated that there is a significant lack of sports facilities in their community. The rare spaces that exist are distant, expensive, or inconvenient. Participants also stated that these spaces are faced with many difficulties, including lack of security, lack of supervision, lack of equipment, harassment (particularly for girls), and poor ventilation for closed spaces. Such problems make these spaces impracticable. Participants also indicated that in the absence of suitable spaces, some adolescents play football in the street. Both girls and parents expressed the lack of spaces for girls. According to participants, addressing the lack of sports facilities is not a priority for local authorities.

Availability and accessibility of spaces (e.g., not distant, unfilled, less expensive, having family income to participate) have been considered significant facilitators of adolescent PA. Some participants stated that there are sufficient places to play football but no space for other types of sports (e.g., basketball, tennis, cycling, and swimming).

\section{Discussion}

By using a qualitative design, our goal was to explore potential barriers and facilitators of adolescent PA. We identified that influencing factors were organized into six themes classified into different levels of the social-ecological model, with three themes belonging to intrapersonal level (perceived motivating and limiting factors, PA awareness, and perceived time constraints), two themes classified into interpersonal/cultural level (social support, and gender and cultural norms), and one theme corresponded to the environmental level (access to opportunities). Such themes include both barriers and facilitators of PA, which have been reported previously $[28,31]$. Interactions between the themes, as shown in the thematic map (Fig. 1), can provide valuable insights to identify possible mechanisms to improve PA. Because reports on interactions and causal pathways within the social-ecological model regarding PA are scarce [45], these findings could be useful in the development of a tailored intervention aimed at improving adolescent PA.

\section{Intrapersonal factors}

As reported previously [25-29, 31], positive expectations in PA, such as having fun, spending time with friends, and breaks to daily routine, were important facilitators of 
adolescent PA. Similarly, all participants considered that health benefits, weight management, and preserving a particular physical appearance were important reasons to engage in PA. These factors were also identified in previous studies [25-28, 31]. In literature, competition is often considered as a barrier to adolescents' PA especially among girls and those lacking skills or physical conditions [25, 26, 28 , 46]. In our study, competition was perceived as a facilitator for PA by some participants, which were almost exclusively boys. A likely explanation for this result may be that these boys have good skills or physical conditions, which encourage them to enjoy physical activities of a competitive nature. Self-defense, against an aggressor for example, was a reason for some adolescents (especially girls) to engage in self-defense sports, expressing feeling unsafe within their neighborhoods. In line with a widely known custom among boys but unusual in the literature, many boys stated that they play football to win money. Hence, organizing sports competitions with prizes to the winning teams could encourage PA in boys.

Similar to previous studies, adolescents with physical disability [47] and/or with poor physical condition and skills $[25,27,28,31,46]$ engage in less PA than their normal peers. In a systematic review, Carlon et al. found that youth with cerebral palsy participated in 13 to $53 \%$ less PA than their peers and were more sedentary [47]. Long-term negative health consequences of inactivity among disabled youth are therefore more likely, which can in turn reduce their life expectancy [47]. Therefore, intervention programs that seek to increase the PA level among this category of population are needed. Interventions can target some modifiable physical, psychological, and environmental correlates identified in previous studies [48]. However, it was concluded in two recent systematic reviews $[49,50]$ that the effectiveness of this kind of interventions was not always sure, and future studies are required before giving recommendations for such interventions. In the literature, physical disability was rarely reported as a barrier, especially in relevant systematic reviews of quantitative studies $[16-18,20]$; this may be because questions related to disabilities were not included in questionnaires measuring PA. Thus, considerations of physical disability in the development of such tools could prevent possible biases in the estimation of PA level.

Similar to a number of previous studies [27-29, 46], our results showed that participation in PA decreases negative psychological characteristics such as low desire and importance, poor enthusiasm toward PA, and laziness, timidity, and fear of accidents. Thus, improvement of these mediators can positively affect adolescent PA level.

Smoking was perceived as a barrier to PA by participants of this study. This behavioral factor was not identified as a barrier in qualitative studies, although results from quantitative studies are inconclusive [16].
Furthermore, in a longitudinal cohort study, Audrain-McGovern et al. concluded that adolescent smoking uptake depended on the kind of PA; some types of PA are negatively associated with smoking uptake, whereas others are positively associated [51]. In studies supporting a negative association between PA and smoking, the underlying mechanisms are not yet understood. Thus, Audrain-McGovern et al. found that PA reward as one mechanism by which PA can reduce the likelihood of adolescent smoking uptake [52]. In the same sense, Verkooijen et al. conducted another study aimed at gaining a better understanding of this association and found that some motives like friendships and competition appeared to moderate the association between PA and smoking rather than others (e.g., weight management and self-esteem). This study recommends focusing on first motives and avoid the last ones when designing a smoking prevention program [53]. Finally, the relationship between PA and smoking is complex, further research is warranted to identify other mechanisms underlying this relationship to provide evidence-based smoking prevention targets [53].

Adolescents and parents were generally aware of the health benefits of PA. In line with previous studies [29, 54], this was considered as a facilitator of PA practice. Regarding adolescent PA guidelines, most adolescents and parents did not know that adolescents must accumulate at least $60 \mathrm{~min}$ of daily moderate to vigorous intensity PA to stay healthy. Being unaware of this threshold may lead to an overestimation of PA level among adolescents, believing themselves to be more active than they really are [55]. In the same way, parents who overestimate the PA level of their children may see no need to encourage them to increase their PA [55]. Improving awareness related to PA health benefits and recommendations among adolescents and their parents may encourage behavior changes in adolescents [55]. Furthermore, involving media, school, and family may be useful in raising PA awareness among adolescents because participants considered them to be the main awareness-raising sources of PA.

Our study results agree with previous findings that lack of time was a strong barrier to adolescent PA [25, 28, 29]. Participants linked the lack of time to school demands (e.g., overloaded curriculum, private lessons, homework, and giving higher priority to academic success), screen device use, time spent with friends, time mismanagement, and household tasks (among girls). Improving the adolescent's time management skills should be targeted when setting up an intervention program. Data from the latest Global School-based Health Survey indicated that $32.9 \%$ of Moroccan adolescents spent 3 or more hours per day on sedentary activities, not including time in school or doing homework [33]. It is proven that sedentary behavior and PA are two different 
constructs and that they do not directly displace one another [56]. Therefore, efforts to encourage decreases in sedentary behavior time and increases in PA must go together [56].

\section{Interpersonal/cultural factors}

Social support has been consistently associated with adolescent PA [21, 57]. This support is provided by different sources (parents, family, friends, and others) and in different ways, either tangibly (e.g., doing activity with, watching/ supervision, transportation, payment of fees) or intangibly (e.g., encouragement and praise, discussing benefits, role models) [20, 30, 57]. Similar to previous studies [28, 29, 58], our findings showed that tangible and intangible social support provided by parents, family, friends, and sometimes by national sports leaders was a salient facilitator of adolescent PA. However, an unsupportive social environment was a barrier to PA. Results also indicated that not having friends or having friends who are physically inactive or with other interests and negative interests (e.g., drug use) may keep adolescents away from PA. Therefore, social support should be targeted in intervention programs aimed at increasing PA levels among adolescents.

Girls are less active than boys, but the reasons behind this are not well understood $[59,60]$. These gender differences were explained previously by non-modifiable variables, including a girl's biology [61], and by some modifiable variables related to a girl's psychology [60, 62, $63]$, social support $[59,60,62]$, or cultural and physical environmental factors $[60,62]$. Our use of single-gender FGs and involvement of parents and teachers proved useful in identifying barriers that led to inactivity in girls. Findings showed a variety of barriers mainly of a sociocultural aspect. Similar to most Arab countries [34, 64], girls received less social support compared with boys, as many families do not allow their girls to practice PA outdoors because of conservative cultural and religious norms. To preserve public modesty, many parents do not permit their girls to practice PA with sports dress, but with traditional dress, which is not suitable for PA [37, 64]. Some traditional long dress covering the whole body may hide women fatness and consequently reduce their motivation to do PA [36, 37, 64]. In addition, cultural expectations concerning women's roles were considered as barriers to PA. Thus, like many countries in the world, Moroccan women were expected to care for the family and household and their exercise needs were afforded low priority $[34,35]$. Participants reported that some sports were socially perceived to be valid only for boys, and there is also a lack of appropriate spaces specific for girls that are secure, safe, and well supervised. In a review on Arab adolescents, Obermeyer and associates indicated that some features of social context such as unequal gender norms can affect adolescent health [38]. Similarly, the recent
Arab Human Development Report indicated that gender equality and women's empowerment are more restricted in Arab regions than in other regions [65]. In brief, the identification of modifiable factors that explained gender differences in adolescent participation in PA is important since they can be targeted in interventions [62]. The mediators found in this study can be targeted to minimize the effects of gender and thus improve PA levels in girls. Nevertheless, objective measurements are required to ensure that they are relevant mediators because previous studies concluded that among a multitude of mediators examined, only some could explain the association between gender and PA $[59,62]$.

\section{Environmental factors}

Various theoretical frameworks have been used to understand factors influencing PA. In the previous decades, the use of ecological models revealed numerous environmental factors that may affect adolescent PA levels. Identification of such factors is useful because young people are especially susceptible to environmental influences given that they have relatively little autonomy over their own behaviors [59]. Furthermore, many studies showed that interventions that affect environmental factors, along with other personal and interpersonal factors, are more likely to be effective [66]. This study showed that PE classes in school and recreational facilities outside school are two opportunities available for adolescents to practice PA.

Similar to previous studies [26, 27], our results highlighted that PE classes contributed to improving the overall PA level among adolescents. However, PE classes face several obstacles related mainly to infrastructure, equipment, schedules, and insufficient hourly volume. In such context, students, especially girls, apply for a certificate of exemption from PE classes. This negative impact of PE classes on PA levels in female adolescents was previously reported by other studies [25, 27]. Consequently, to improve adolescent PA, PE classes must be reinforced because it touches a high number of adolescents regardless of their social differences.

Outside school, participants stated that there is a lack of recreational facilities for many sports (e.g., jogging, cycling, basketball, tennis, swimming). The rare existing spaces, for football mainly, were often distant, insecure, unsupervised, not equipped, or expensive, often making these spaces impracticable. These issues were more pronounced among girls. According to the participants, a lack of opportunities outside the school was because addressing these issues was not a priority for city policymakers.

Participant recruitment in this study was limited to two middle schools in an urban area. Thus, it is not possible to generalize findings to a larger population. 
However, our goal was to generate a deep exploration of influencing factors of adolescent PA and not to generalize results. However, our high number of participants, as well as triangulation of participants, allowed us to obtain a more complete overview. Because of the qualitative nature of this research, results are not equal to objectively measured data. Thus, it was not possible to define which influencing factor was most significant for adolescent participation in PA. Furthermore, we did not give the percentages of participants reporting a theme or subtheme. As reported by Braun and Clarke [22], the issue raised most frequently or by a larger number of participants is not necessarily the most pertinent to answer the research question. Finally, the interactions between themes, as shown in the thematic map (Fig. 1), require an objective evaluation to prove their strength.

\section{Conclusions}

This qualitative study is the first in Morocco to explore the identification of barriers and facilitators of adolescent PA. Overall, our findings show that barriers and facilitators are organized into six themes (perceived motivating and limiting factors, PA awareness and perceived time constraints, social support, gender and cultural norms, and access to opportunities). These themes included both barriers and facilitators of PA and belonged to different levels of the social-ecological model. Our findings have several implications. First, they could be used to develop tailored intervention programs aimed at improving adolescent PA. Second, they can improve quantitative research by enriching the battery of questions of PA instruments (e.g., a question related to physical disability). Third, the proposed thematic map (Fig. 1) can contribute to understanding interactions and causal pathways in the social-ecological model. However, these interactions require an objective evaluation to prove their strength.

\section{Abbreviations}

FG: Focus group; PA: Physical activity; PE: Physical education

\section{Acknowledgements}

We are very grateful to all participants (adolescents, parents and teachers) who took part in FG discussions. We also thank the provincial director of Education Ministry and teachers/ headmasters/principals of the participating schools for their invaluable collaboration. Special thanks are due to Rasa G. Hamilton (Moffitt Cancer Center) for her generous assistance with this publication. Our thanks also go to Azzam H. and her team for the workshops organized in Morocco on capacity building where this project has matured. We would also like to thank El Rhazi K. for the coordination of these workshops.

\section{Funding}

This study is supported by Moffitt Cancer Centre under the NIH International Fogarty Centre for the award number 5D43TW009804 (PI Anna Giuliano).

\section{Availability of data and materials}

All datasets generated and/or analyzed during the current study are not publicly available due to their personal nature but are available from the corresponding author on reasonable request.

\section{Authors' contributions}

AE carried out the literature searches and drafted and finalized the paper. All authors contributed equally in designing the study. $A E, H E$, and SB were responsible for the data collection, transcription, coding, and analysis. YE and SE helped in the data coding and analysis, supervised the study, and coordinated the research team. All authors have read, revised and approved the final draft of the manuscript.

\section{Ethics approval and consent to participate}

The study was approved by the Faculty of Medicine and Pharmacy of Casablanca Research Ethics Committee and by the National Control Commission for the Protection of Personal Data (A-RS-193-2015) and authorized by The Provincial Directorate of Education Ministry in Taza. Informed consent was obtained from all parents and teachers. Adolescent consent forms were signed by their parents, but an additionally verbal consent was also given by adolescent participants.

\section{Consent for publication}

Not applicable.

\section{Competing interests}

The authors declare that they do not have competing interests.

\section{Publisher's Note}

Springer Nature remains neutral with regard to jurisdictional claims in published maps and institutional affiliations.

\section{Author details}

${ }^{1}$ Laboratory of Epidemiology, Clinical Research and Community Health, Faculty of Medicine and Pharmacy, University Sidi Mohamed Ben Abdellah, Fez, Morocco. ${ }^{2}$ Regional Centre for Careers Education and Training (CRMEF Fès-Meknes), Fez, Morocco.

Received: 27 November 2018 Accepted: 28 March 2019

Published online: 09 April 2019

\section{References}

1. Blakemore SJ, Mills KL. Is adolescence a sensitive period for sociocultural processing. Annu Rev Psychol. 2013;65:187-07.

2. Sawyer SM, Afifi RA, Bearinger LH, Blakemore SJ, Dick B, Ezeh AC, et al. Adolescence: a foundation for future health. Lancet. 2012;379:1630-40.

3. Patton GC, Sawyer SM, Santelli JS, Ross DA, Afifi R, Allen NB, et al. Our future: a lancet commission on adolescent health and wellbeing. Lancet. 2016;387:2423-78

4. Dumith SC, Gigante DP, Domingues MR, Kohl HW. Physical activity change during adolescence: a systematic review and a pooled analysis. Int J Epidemiol. 2011;40:685-98.

5. Lee IM, Shiroma EJ, Lobelo F, Puska P, Blair SN, Katzmarzyk PT. Effect of physical inactivity on major non-communicable diseases worldwide: an analysis of burden of disease and life expectancy. Lancet. 2012;380:219-29.

6. Committee WGAbtGR. Global recommendations on physical activity for health. 2015/07/17 edition; 2010.

7. Gaba A, Mitas J, Jakubec L. Associations between accelerometer-measured physical activity and body fatness in school-aged children. Environ Health Prev Med. 2017;22:43.

8. Spruit A, Assink M, van Vugt E, van der Put C, Stams GJ. The effects of physical activity interventions on psychosocial outcomes in adolescents: a meta-analytic review. Clin Psychol Rev. 2016;45:56-71.

9. Hyakutake A, Kamijo T, Misawa Y, Washizuka S, Inaba Y, Tsukahara T, et al. Cross-sectional observation of the relationship of depressive symptoms with lifestyles and parents' status among Japanese junior high school students. Environ Health Prev Med. 2016;21:265-73.

10. Poitras VJ, Gray CE, Borghese MM, Carson V, Chaput JP, Janssen I, et al. Systematic review of the relationships between objectively measured physical activity and health indicators in school-aged children and youth. Appl Physiol Nutr Metab. 2016;41:S197-39. 
11. Janssen I, Leblanc AG. Systematic review of the health benefits of physical activity and fitness in school-aged children and youth. Int J Behav Nutr Phys Act. 2010;7:40.

12. de Moraes AC, Guerra PH, Menezes PR. The worldwide prevalence of insufficient physical activity in adolescents; a systematic review. Nutr Hosp. 2013;28:575-84

13. Itoh H, Kitamura F, Hagi N, Mashiko T, Matsukawa T, Yokoyama K. Leisure time physical activity in youth as a predictor of adult leisure physical activity among Japanese workers: a cross-sectional study. Environ Health Prev Med. 2017:22:37.

14. Sallis JF, Owen N, Fotheringham MJ. Behavioral epidemiology: a systematic framework to classify phases of research on health promotion and disease prevention. Ann Behav Med. 2000;22:294-8.

15. Sallis JF, Cervero RB, Ascher W, Henderson KA, Kraft MK, Kerr J. An ecological approach to creating active living communities. Annu Rev Public Health. 2006;27:297-22.

16. Bauman AE, Reis RS, Sallis JF, Wells JC, Loos RJ, Martin BW. Correlates of physical activity: why are some people physically active and others not. Lancet. 2012;380:258-71.

17. Craggs C, Corder K, van Sluijs EM, Griffin SJ. Determinants of change in physical activity in children and adolescents: a systematic review. Am J Prev Med. 2011;40:645-58.

18. Van Der Horst K, Paw MJ, Twisk JW, Van Mechelen W. A brief review on correlates of physical activity and sedentariness in youth. Med Sci Sports Exerc. 2007;39:1241-50

19. de Vet E, de Ridder DT, de Wit JB. Environmental correlates of physical activity and dietary behaviours among young people: a systematic review of reviews. Obes Rev. 2011;12:e130-42.

20. Ferreira I, van der Horst K, Wendel-Vos W, Kremers S, van Lenthe FJ, Brug J. Environmental correlates of physical activity in youth - a review and update. Obes Rev. 2007:8:129-54

21. Laird Y, Fawkner S, Kelly P, McNamee L, Niven A. The role of social support on physical activity behaviour in adolescent girls: a systematic review and meta-analysis. Int J Behav Nutr Phys Act. 2016;13:79.

22. Clarke $V$, Braun V. Successful qualitative research: a practical guide for beginners. London: Sage; 2013.

23. Zitomer MR, Goodwin D. Gauging the quality of qualitative research in adapted physical activity. Adapt Phys Act Q. 2014;31:193-18.

24. Pitney WA, Parker J. Qualitative research in physical activity and the health professions. Champaign: Human Kinetics; 2009

25. Allender S, Cowburn G, Foster C. Understanding participation in sport and physical activity among children and adults: a review of qualitative studies. Health Educ Res. 2006;21:826-35.

26. Casey MM, Eime RM, Payne WR, Harvey JT. Using a socioecological approach to examine participation in sport and physical activity among rural adolescent girls. Qual Health Res. 2009;19:881-93.

27. Hohepa M, Schofield G, Kolt GS. Physical activity: what do high school students think. J Adolesc Health. 2006;39:328-36.

28. Martins J, Marques A, Sarmento H, Carreiro da Costa F. Adolescents' perspectives on the barriers and facilitators of physical activity: a systematic review of qualitative studies. Health Educ Res. 2015;30:742-55.

29. Van Royen K, Verstraeten R, Andrade S, Ochoa-Aviles A, Donoso S, Maes L, et al. Factors affecting physical activity in Ecuadorian adolescents: a focus group study. J Phys Act Health. 2015;12:340-8.

30. Beets MW, Cardinal BJ, Alderman BL. Parental social support and the physical activity-related behaviors of youth: a review. Health Educ Behav. 2010;37:621-44

31. Belanger M, Casey M, Cormier M, Filion AL, Martin G, Aubut S, et al. Maintenance and decline of physical activity during adolescence: insights from a qualitative study. Int J Behav Nutr Phys Act. 2011;8:117.

32. Guthold R, Cowan MJ, Autenrieth CS, Kann L, Riley LM. Physical activity and sedentary behavior among schoolchildren: a 34-country comparison. J Pediatr. 2010;157:43-9.

33. CDC. Global School-based Health Survey. Available at: https://www.cdc.gov/ gshs/countries/eastmediter/morocco.htm. Accessed 01 Aug 2016.

34. Sharara E, Akik C, Ghattas H, Makhlouf Obermeyer C. Physical inactivity, gender and culture in Arab countries: a systematic assessment of the literature. BMC Public Health. 2018:18:639.

35. Benjamin K, Donnelly $\Pi$. Barriers and facilitators influencing the physical activity of Arabic adults: a literature review. Avicenna. 2013:8. https://doi. org/10.5339/avi.2013.8
36. Musaiger AO. Overweight and obesity in eastern mediterranean region: prevalence and possible causes. J Obes. 2011:2011:407237.

37. Rguibi M, Belahsen R. Body size preferences and sociocultural influences on attitudes towards obesity among Moroccan Sahraoui women. Body Image. 2006;3:395-400.

38. Obermeyer CM, Bott S, Sassine AJ. Arab adolescents: health, gender, and social context. J Adolesc Health. 2015;57:252-62

39. High Commission for Planning (HCP). Available at: http://www.hcp.ma/. Accessed 01 Feb 2016

40. Santiago-Delefosse M, Gavin A, Bruchez C, Roux P, Stephen SL. Quality of qualitative research in the health sciences: analysis of the common criteria present in 58 assessment guidelines by expert users. Soc Sci Med. 2016;148:142-51.

41. El Achhab Y, El Ammari A, El Kazdouh H, Najdi A, Berraho M, Tachfouti N, Lamri D, El Fakir S, Nejjari C. Health risk behaviours amongst school adolescents: protocol for a mixed methods study. BMC Public Health. 2016;16:1209.

42. El-ammari A, El kazdouh H, Bouftini S, El fakir S, El achhab Y. Level and potential social-ecological factors associated with physical inactivity and sedentary behavior among Moroccan school-age adolescents: a crosssectional study. Environ Health Prev Med. 2017:22:47.

43. Morgan DL. Qualitative research methods: focus groups as qualitative research. $2^{\text {nd }}$ ed: SAGE Publications; 1997. https://doi.org/10.4135/ 9781412984287

44. Braun V, Clarke V. Using thematic analysis in psychology. Qual Res Psychol. 2006;3(2):77-101.

45. Glanz K, Rimer BK, Viswanath K. Health behavior and health education: theory, research, and practice. 4th ed. San Francisco: Wiley; 2008.

46. Jonsson L, Berg C, Larsson C, Korp P, Lindgren EC. Facilitators of physical activity: voices of adolescents in a disadvantaged community. Int J Environ Res Public Health. 2017;14:839.

47. Carlon SL, Taylor NF, Dodd KJ, Shields N. Differences in habitual physical activity levels of young people with cerebral palsy and their typically developing peers: a systematic review. Disabil Rehabil. 2013:35:647-55.

48. Li R, Sit CHP, Yu JJ, Duan JZJ, Fan TCM, McKenzie TL, et al. Correlates of physical activity in children and adolescents with physical disabilities: a systematic review. Prev Med. 2016;89:184-93.

49. McGarty AM, Downs SJ, Melville CA, Harris L. A systematic review and metaanalysis of interventions to increase physical activity in children and adolescents with intellectual disabilities. J Intellect Disabil Res. 2018;62:312-29.

50. Hassan NM, Landorf KB, Shields N, Munteanu SE. Effectiveness of interventions to increase physical activity in individuals with intellectual disabilities: a systematic review of randomised controlled trials. J Intellect Disabil Res. 2019;63:168-91.

51. Audrain-McGovern J, Rodriguez D. All physical activity may not be associated with a lower likelihood of adolescent smoking uptake. Addict Behav. 2015;51:177-83.

52. Audrain-McGovern J, Rodriguez D, Cuevas J, Sass J. Initial insight into why physical activity may help prevent adolescent smoking uptake. Drug Alcohol Depend. 2013;132:471-8.

53. Verkooijen KT, Nielsen GA, Kremers SPJ. Leisure time physical activity motives and smoking in adolescence. Psychol Sport Exerc. 2009;10:559-64

54. Goh YY, Bogart LM, Sipple-Asher BK, Uyeda K, Hawes-Dawson J, OlaritaDhungana J, et al. Using community-based participatory research to identify potential interventions to overcome barriers to adolescents' healthy eating and physical activity. J Behav Med. 2009;32:491-02.

55. Vanhelst J, Beghin L, Duhamel A, De Henauw S, Ruiz JR, Kafatos A, et al. Physical activity awareness of European adolescents: the HELENA study. J Sports Sci. 2018;36:558-64.

56. Pearson N, Braithwaite RE, Biddle SJ, van Sluijs EM, Atkin AJ. Associations between sedentary behaviour and physical activity in children and adolescents: a meta-analysis. Obes Rev. 2014;15:666-75.

57. Mendonca G, Cheng LA, Melo EN, de Farias Junior JC. Physical activity and social support in adolescents: a systematic review. Health Educ Res. 2014;29:822-39.

58. Edwardson $\mathrm{CL}$, Gorely T. Parental influences on different types and intensities of physical activity in youth: a systematic review. Psychol Sport Exerc. 2010;11:522-35

59. Crespo NC, Corder K, Marshall S, Norman GJ, Patrick K, Sallis JF, et al. An examination of multilevel factors that may explain gender differences in children's physical activity. J Phys Act Health. 2013:10:982-92.

60. Spencer RA, Rehman L, Kirk SF. Understanding gender norms, nutrition, and physical activity in adolescent girls: a scoping review. Int J Behav Nutr Phys Act. 2015;12:6 
61. Maia JA, Thomis M, Beunen G. Genetic factors in physical activity levels: a twin study. Am J Prev Med. 2002;23:87-91.

62. Patnode CD, Lytle LA, Erickson DJ, Sirard JR, Barr-Anderson D, Story M. The relative influence of demographic, individual, social, and environmental factors on physical activity among boys and girls. Int J Behav Nutr Phys Act. 2010;7:79.

63. Spence JC, Blanchard CM, Clark M, Plotnikoff RC, Storey KE, McCargar L. The role of self-efficacy in explaining gender differences in physical activity among adolescents: a multilevel analysis. J Phys Act Health. 2010;7:176-83.

64. Musaiger AO, Al-Mannai M, Tayyem R, Al-Lalla O, Ali EY, Kalam F, et al. Perceived barriers to healthy eating and physical activity among adolescents in seven Arab countries: a cross-cultural study. ScientificWorld J. 2013;2013:232164.

65. UNDP. Youth and the prospects for human development in a changing reality: Arab human development report 2009. New York: United Nations development Programme (UNDP), Regional Bureau for Arab States (RBAS); 2016.

66. Heath GW, Parra DC, Sarmiento OL, Andersen LB, Owen N, Goenka S, et al. Evidence-based intervention in physical activity: lessons from around the world. Lancet. 2012;380:272-81.

Ready to submit your research? Choose BMC and benefit from:

- fast, convenient online submission

- thorough peer review by experienced researchers in your field

- rapid publication on acceptance

- support for research data, including large and complex data types

- gold Open Access which fosters wider collaboration and increased citations

- maximum visibility for your research: over $100 \mathrm{M}$ website views per year

At $\mathrm{BMC}$, research is always in progress.

Learn more biomedcentral.com/submissions 